\title{
Anticonvulsant and Antiepileptogenic Effects Mediated by Adeno-Associated Virus Vector Neuropeptide Y Expression in the Rat Hippocampus
}

\author{
Cristina Richichi, ${ }^{1 \star}$ En-Ju D. Lin, ${ }^{2 *}$ Daniela Stefanin, ${ }^{1}$ Daniele Colella, ${ }^{1}$ Teresa Ravizza, ${ }^{1}$ Giuliano Grignaschi, ${ }^{1}$ \\ Pietro Veglianese, ${ }^{1}$ Günther Sperk, ${ }^{3}$ Matthew J. During, ${ }^{2}$ and Annamaria Vezzani ${ }^{1}$ \\ ${ }^{1}$ Department of Neuroscience, Mario Negri Institute for Pharmacological Research, 20157 Milan, Italy, ${ }^{2}$ Department of Molecular Medicine, The University \\ of Auckland, Auckland 1020, New Zealand, and ${ }^{3}$ Department of Pharmacology, University of Innsbruck, 6020 Innsbruck, Austria
}

Neuropeptide Y (NPY) inhibits seizures in experimental models and reduces excitability in human epileptic tissue. We studied the effect of long-lasting NPY overexpression in the rat hippocampus with local application of recombinant adeno-associated viral (AAV) vectors on acute kainate seizures and kindling epileptogenesis.

Transgene expression was significantly increased by $7 \mathrm{~d}$, reached maximal expression by 2 weeks, and persisted for at least 3 months. Serotype $2 \mathrm{AAV}$ vector increased NPY expression in hilar interneurons, whereas the chimeric serotype 1/2 vector caused far more widespread expression, also including mossy fibers, pyramidal cells, and the subiculum. EEG seizures induced by intrahippocampal kainate were reduced by $50-75 \%$, depending on the vector serotype, and seizure onset was markedly delayed.

In rats injected with the chimeric serotype $1 / 2$ vector, status epilepticus was abolished, and kindling acquisition was significantly delayed. Thus, targeted NPY gene transfer provides a potential therapeutic principle for the treatment of drug-resistant partial epilepsies.

Key words: epilepsy; fascia dentata; gene; kainic acid; kindling; seizure

\section{Introduction}

Neuropeptide Y (NPY), a 36 aa polypeptide, is widely distributed in the CNS, where it is involved in various physiological functions (Mitchell et al., 1997; Pedrazzini et al., 2003). Recently, NPY has become the focus of much attention for its possible involvement in epilepsy (Redrobe et al., 1999; Vezzani et al., 1999a). Despite its widespread distribution in the brain, changes in NPY and its receptors occur in brain regions crucially involved in initiation and propagation of seizures. In rodents, seizures enhance NPY levels in hilar inhibitory hippocampal interneurons, where this peptide is expressed in physiological conditions, and in the excitatory granule cells and their mossy fibers that do not normally contain NPY (Marksteiner et al., 1990; for review, see Vezzani et al., 1999a).

In the hippocampi of patients with intractable temporal lobe epilepsy (TLE), NPY-overexpressing interneurons sprout into terminal areas of excitatory perforant path axons and mossy fiber terminals (de Lanerolle et al., 1989; Mathern et al., 1995; Furtinger et al., 2001).

\footnotetext{
Received Sept. 3, 2003; revised Jan. 19, 2004; accepted Jan. 19, 2004

This work was supported by the Human Frontier Science Program (RG0045/2000-B) (A.V., G.S.), Fondazione Cariplo (A.V.), and the New Zealand Health Research Council (M.J.D.). We thank Daniela Moneta and Eleonora Zennaro for contributing to part of this work.

${ }^{*}$ C.R. and E.-J.D.L. contributed equally to this study.

Correspondence should be addressed to Dr. Annamaria Vezzani, Laboratory of Experimental Neurology, Department of Neuroscience, Mario Negri Institute for Pharmacological Research, Via Eritrea 62, 20157 Milan, Italy. E-mail: Vezzani@marionegri.it.

DOI:10.1523/JNEUROSCI.4056-03.2004

Copyright $\odot 2004$ Society for Neuroscience $\quad$ 0270-6474/04/243051-09\$15.00/0
}

These plastic changes may subserve inhibitory effects on neuronal excitability and consequently on seizures. Thus, NPY overexpression in hippocampal GABA-containing interneurons could lead to increased inhibitory input onto terminals of pyramidal neurons and granule cells (Milner et al., 1997). This action is likely mediated by presynaptic NPY-Y2 receptors inhibiting glutamate release (Colmers and Bleakman, 1994; Greber et al., 1994), and Y2 receptors are upregulated at these sites in epileptic tissue from experimental models and TLE patients (for review, see Vezzani et al., 1999a; Furtinger et al., 2001). Evidence for an inhibitory effect on seizures mediated by Y5 receptors has also been reported (Marsh et al., 1999), and both upregulation and downregulation of these receptors have been found in brain tissue after experimentally induced seizures (Kopp et al., 1999; Bregola et al., 2000; Vezzani et al., 2000).

Compelling evidence supports an anticonvulsant role for NPY, both when exogenously applied and when endogenously released. Thus, transgenic rats overexpressing NPY showed reduced seizure susceptibility and epileptogenesis (Vezzani et al., 2002), whereas knock-out mice lacking NPY or the Y2 or Y5 receptor gene are more vulnerable to chemically or electrically induced convulsions (Baraban et al., 1997; Marsh et al., 1999; DePrato Primeaux et al., 2000; Weinshenker et al., 2001; El Bahh et al., 2002). In hippocampal slices from epileptic patients with hippocampal seizure onset, NPY has potent and long-lasting inhibitory action on perforant path-evoked excitatory responses from dentate granule cells (Patrylo et al., 1999). Thus, we hypothesized that augmentation of local inhibitory tone resulting from 
neuronal overexpression of NPY in the hippocampus using a recombinant adeno-associated viral (rAAV) vector may be an effective strategy for inhibition of seizures and epileptogenesis. rAAV vector can successfully transduce postmitotic cells, such as mature neurons, with high efficiency and stability, minimal immunogenicity and nonpathogenicity, and broad host and cell range (Kaplitt et al., 1994; During, 1997; During et al., 2003). In this study, we constructed an rAAV vector designed to express NPY in neurons.

We found that rats with hippocampal NPY overexpression had a dramatic reduction in kainic acid-induced EEG seizures, a delay in their onset, and impaired kindling epileptogenesis. These data represent a proof of principle of the applicability of gene transfer for novel antiepileptogenic strategies.

\section{Materials and Methods}

Experimental animals. Male Sprague Dawley adult rats $(\sim 220 \mathrm{gm}$; Charles River, Calco, Italy) were used. They were housed at a constant temperature $\left(23^{\circ} \mathrm{C}\right)$ and relative humidity $(60 \%)$ with a fixed $12 \mathrm{hr}$ light/ dark cycle and ad libitum access to food and water. Procedures involving animals and their care were conducted in conformity with the institutional guidelines that are in compliance with national and international laws and policies.

Plasmid construction and AAV vector production. The plasmid rAAVneuron-specific enolase (NSE)-NPY woodchuck post-translational regulatory element (WPRE) (rAAV-NPY) was constructed using standard molecular cloning procedures. Human prepro-NPY (ppNPY) cDNA was subcloned into an expression cassette consisting of the rat NSE promoter, WPRE, and a bovine growth hormone poly(A) signal. Enhanced green fluorescent protein (GFP) (Clontech, Palo Alto, CA) was subcloned into pAM-NSE-pl-WPRE simian virus 40 poly(A), as described in detail previously (Xu et al., 2001). The same expression cassette without the transgene was used as a control (rAAV-empty). Expression cassettes were subcloned into the rAAV backbone, which was flanked by rAAV2 inverted terminal repeats.

High-titer rAAV serotype 2 vectors were generated by packaging the rAAV plasmids, as described previously (During et al., 2003). Chimeric rAAV1-AAV2 vectors were packaged using the identical published protocol with the addition of an rAAV1 capsid helper plasmid in a 1:1 ratio with the rAAV2 helper; the presence of rAAV2 capsid proteins retained the heparin-binding domain and allowed column purification. Genomic titers of vector stocks were determined using the Prism 7700 sequence detector system (PerkinElmer Applied Biosystems, Foster City, CA), as described previously (Clark et al., 1999).

NPY gene delivery. Two different $\mathrm{rAAV}-\mathrm{NSE}-\mathrm{NPY}$ vectors were used: either serotype 2 or the chimeric serotype containing both rAAV1 and rAAV2 capsid proteins. rAAV vector was injected bilaterally into the dorsal or the dorsal and ventral aspects of the rat hippocampus (the total volume infused in each site was $3 \mu \mathrm{l}$ of rAAV-NSE-NPY of a stock solution of $1.06 \times 10^{12}$ number of particles per milliliter). A total of $3 \mu \mathrm{l}$ was infused in 45 min using a $10 \mu$ l Hamilton syringe and infusion pump controlled by a microprocessor, as described previously (Mastakov et al., 2002; Lin et al., 2003). For rats subjected to subsequent intrahippocampal kainic acid injections, rAAV serotype $2(n=5$ rats) or serotype $1 / 2$ ( $n=10$ rats) was infused bilaterally into the dorsal hippocampus only. For rats subjected to subsequent intraventricular kainic acid injection $(n=6)$ or kindled in the ventral hippocampus $(n=7)$, serotype $1 / 2$ vector was injected bilaterally into both dorsal (nose bar, $-2.5 \mathrm{~mm}$; anteroposterior (AP), -3.1 ; lateral (L), $\pm 2.0 \mathrm{~mm}$ from bregma and 4.2 $\mathrm{mm}$ below dura) and ventral (nose bar, $2.5 \mathrm{~mm}$; AP, $-6.2 \mathrm{~mm}$; L, \pm 4.5 $\mathrm{mm}$ from bregma and $6.0 \mathrm{~mm}$ below dura) aspects of the hippocampus. These multiple injections were done in an attempt to obtain maximal hippocampal-mediated inhibition on seizures and their spread when studying models in which seizures generalize to extrahippocampal regions. All injections were made under stereotactic guidance in Equithesin ( $1 \%$ pentobarbital-4\% chloral hydrate)-anesthetized rats $(3.5 \mathrm{ml} / \mathrm{kg}$, i.p.) (Vezzani et al., 1999b). Control rats ( $n=5-10$ in each group) were injected with equivalent volumes of rAAV-NSE-empty.
The weight of the rats injected with the vectors and overexpressing NPY in the hippocampus did not differ from their age-matched controls (data not shown). We did not observe any obvious changes in the behavior of the rats overexpressing NPY; however, this aspect has not been carefully investigated in this study.

Kainic acid. Eight weeks after either rAAV-NSE-NPY or rAAV-empty injection, rats were implanted under deep Equithesin anesthesia with hippocampal and cortical electrodes and guide cannulas for drug injection, as described previously (Vezzani et al., 1999b, 2002). Four days after surgery, freely moving rats were unilaterally injected with kainic acid into the dorsal hippocampus ( $40 \mathrm{ng} ; n=5-10$ ) or the lateral ventricle (250 ng; $n=6$ ). EEG recordings were made before (baseline) as well as during and up to 180 min after kainic acid and were analyzed visually to detect any activity different from baseline by an investigator blinded to the identity of the rats. EEG seizures consisted of the simultaneous occurrence of high-frequency and multispike complexes, and high-voltage synchronized spike or wave activity in cortical and hippocampal leads of recording (Vezzani et al., 1999b, 2002). EEG seizure activity was quantified by measuring the latency to the first seizure (onset), the number of seizures, and their duration in the $3 \mathrm{hr}$ of recording. This experimental model of seizures was chosen because it is highly sensitive to the modulatory effects of NPY receptor ligands (Vezzani et al., 2000).

Rapid kindling. A different group of rats injected 8 weeks previously with rAAV-empty vector $(n=9)$ or chimeric $1 / 2$ rAAV-NSE-NPY $(n=$ 7) was electrically stimulated and EEG recorded in the left ventral hippocampus according to a well established rapid kindling protocol (Kopp et al., 1999) using constant current stimuli $(50 \mathrm{~Hz} ; 10 \mathrm{sec}$ trains of 400 $\mu \mathrm{A} ; 1 \mathrm{msec}$ bipolar square waves) through a bipolar electrode with a 5 min interval for $200 \mathrm{~min}$. Behavior was observed and scored according to Racine (1972). Afterdischarges were measured in the stimulated hippocampus after each stimulation for every animal. Twenty-four hours after kindling completion, fully kindled rats received five additional electrical stimulations (retest day), as described above, to confirm kindling maintenance.

In situ hybridization analysis of NPY $m R N A$. Rats that were not subjected to seizures were injected with rAAV-NSE-NPY (serotype 2 or 1/2) or rAAV-empty vector $(n=3-4)$ and killed by decapitation 8 weeks later; their brains were rapidly frozen in isopentane $\left(-70^{\circ} \mathrm{C}\right)$. Coronal sections $(20 \mu \mathrm{m})$ were cryosectioned, mounted on gelatin-coated slides, and stored at $-30^{\circ} \mathrm{C}$. In situ hybridization for NPY mRNA was done as described in detail previously (Gruber et al., 1994). A 46 mer oligonucleotide probe (CTCTGTCTGGTGATGAGATTGATGTAGTGTCGCAGAGCGGAGTAGT) complementary to bases 214-259 (GenBank accession number NM_012614) of ppNPY mRNA was used and radiolabeled with $\left[\alpha{ }^{35}\right.$ S $]$ thio-deoxy-ATP $(1300 \mathrm{Ci} / \mathrm{mmol}$; NEN, Wilmington, DE) on their $3^{\prime}$ end by reaction with terminal deoxynucleotidyltransferase (Roche Diagnostics, Mannheim, Germany).

After incubation at $42^{\circ} \mathrm{C}(18 \mathrm{hr})$ and stringent washing, sections were first exposed to BioMax MR films (Amersham Biosciences, Buckinghamshire, UK) for $3 \mathrm{~d}$, subsequently dipped in NTB-2 photosensitive emulsion (diluted 1:1 with distilled water; Eastman Kodak, Rochester, NY), and then exposed for 1 week. After developing the photoemulsion and counterstaining with cresyl violet, the sections were coverslipped.

Quantification of the hybridization signal was done in the hilar region of serotype 2 vector-injected rats according to a method described in detail previously by Masseroli et al. (1993). Briefly, two consecutive slices were evaluated from each rat brain injected with serotype 2 rAAV-NSENPY ( $n=4$ rats) or empty vector $(n=3$ rats). Slices from experimental and control rats were matched at the same anteroposterior level, and in situ hybridization was done simultaneously. Digital images of the slices were used to divide the hilar region (as defined by the triangular-shaped area comprised between the upper and lower blades of the dentate gyrus) in four acquisition frames of $631.04 \mu \mathrm{m}^{2}$ each. The limited number of NPY mRNA-positive cells in the dentate gyrus allows a complete count of every single positive cell within the area. Quantification is based on the real size and gray level of a single grain obtained by computerized microscope image analysis (IBAS 2, PC 286; Kontron-Zeiss, Oberkochen, Germany). The program provides for automatic identification of cell area, the portion occupied by grains, and their gray level. The number of grains 

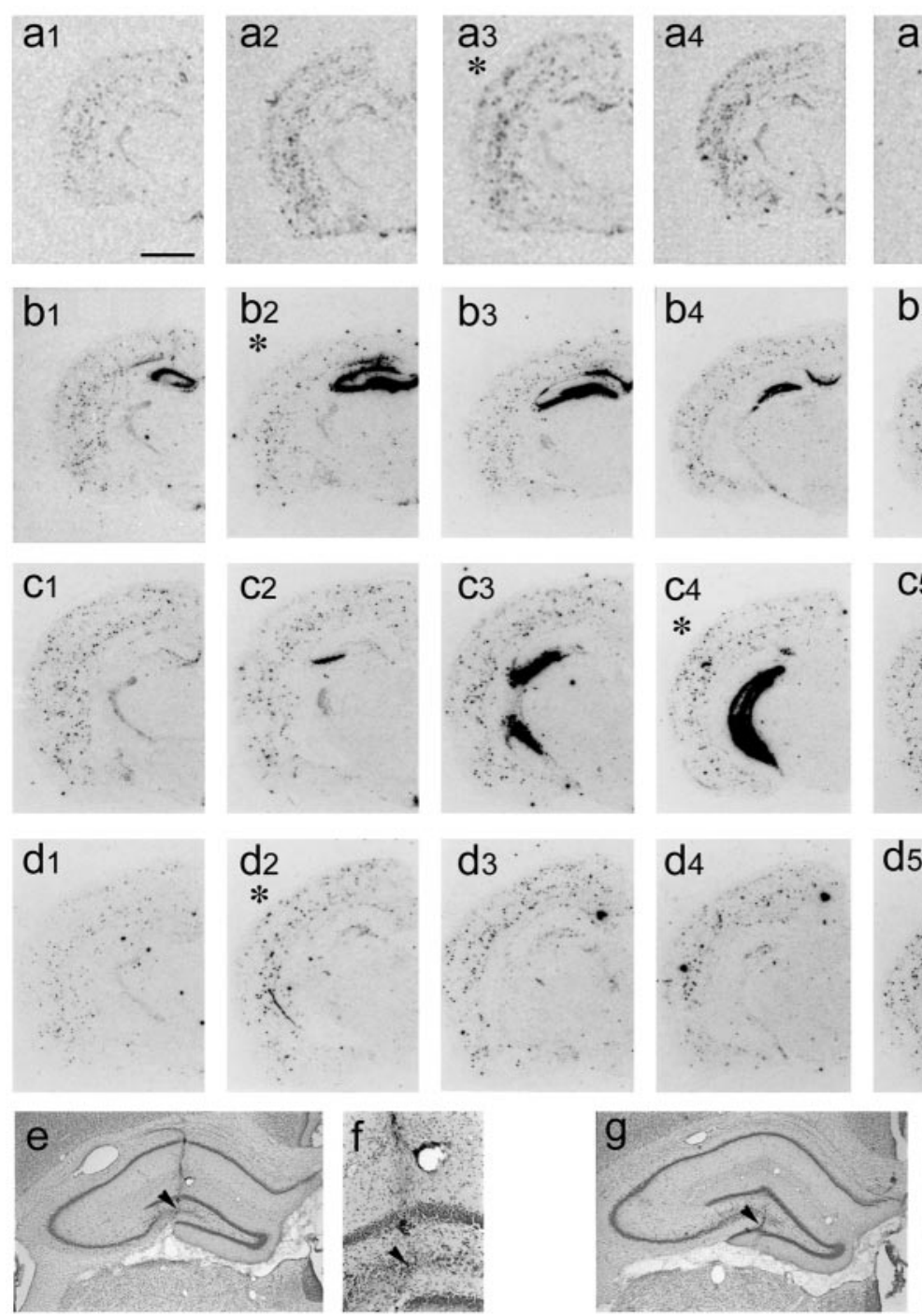

Figure 1. Anteroposterior spread of NPY expression in the injected hippocampus of representative rats 8 weeks after infusion of serotype $2(a 1-a 5)$ or serotype $1 / 2$ rAAV-NSE-NPY $(b 1-b 5, c 1-c 5)$ or in empty vector-injected rats (d1-d5). For serotype 2 rAAV-NSE-NPY, a1-a5 depict the hybridization signal in anteroposterior representative slices encompassing the injection site. In a3 (marked with an asterisk), the signal was higher in the dentate gyrus compared with a matched slice from empty vectorinjected rats (d2). a1 and $a 5$ show a hybridization signal in the dentate gyrus similar to matched slices from controls ( $d 1, d 4$, respectively). High-magnification micrographs showing the hybridization signal in the dentate gyrus of representative experimental and control rats are shown in Figure 2. The spread of the vector around the injected site was $\sim 1.5 \mathrm{~mm}$. For serotype $1 / 2$ rAAV-NSE-NPY, b1-c5 depict the hybridization signal in anteroposterior representative slices encompassing the dorsal (b1-b5) or ventral $(c 1-c 5)$ injected hippocampus. 62 and $c 4$ (marked by asterisks) depict the slices closest to the site of the injection. High-magnification pictures of NPY mRNA expression in representative rats injected with serotype $1 / 2$ are shown in Figure 3 . The spread of this vector around the injection site was $\sim 2.5 \mathrm{~mm} . e-h$ show Nissl-stained sections from rats injected 8 weeks previously with rAAV-NSE-NPY serotype $2(e, f)$ or serotype $1 / 2(g, h)$. $e$ and $g$ depict the dentate gyrus and high magnification of the polymorphic cell layer of the dentate hilus close to the injection site ( $f, h$, arrowheads). We observed only mechanical damage attributable to insertion of the needle track similar to that found in empty vector- or saline-injected rats (data not shown). Scale bars: $a 1-d 5,800 \mu \mathrm{m} ; e-h, 500 \mu \mathrm{m}$.

per cell results from a mathematical function integrating this parameter with a "density factor" (Masseroli et al., 1993). Background is evaluated on the basis of the average cell area $\left(144 \pm 32 \mu \mathrm{m}^{2}\right)$ occupied by grains in regions without a specific hybridization signal (i.e., the stratum moleculare). The measures were made by an operator who was unaware of the section identity. The values obtained in the corresponding acquisition frames in two slices from the same rat were averaged and used for statistical analysis.

Immunohistochemistry. Rats that were not subjected to seizures were injected with rAAV-NSE-NPY (serotype 2 or $1 / 2$ ) or rAAV-empty vector $(n=3$ rats each group) and killed $3 \mathrm{~d}$ to 3 months later by transcardial
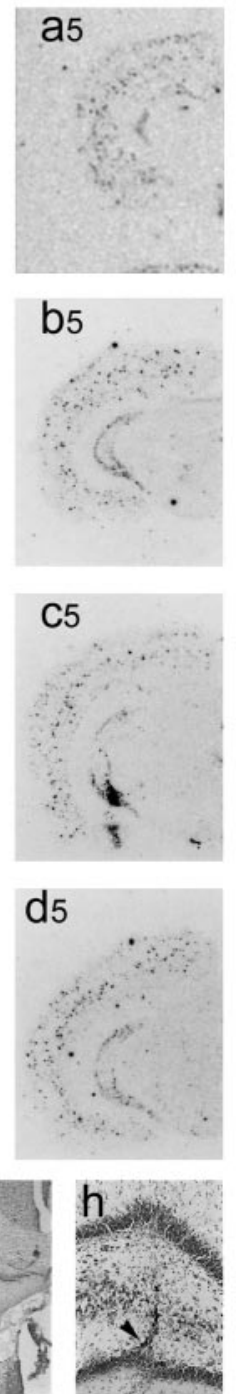

perfusion with PBS followed by $4 \%$ paraformaldehyde in PBS under Equithesin anesthesia (Vezzani et al., 2002). Their brains were postfixed in the same fixative overnight at $4^{\circ} \mathrm{C}$ and then cryoprotected in 30\% sucrose in PBS. Forty micrometer coronal hippocampal sections were cryosectioned in each rat through the entire septotemporal extension, and every fifth section was used. Immunohistochemistry was performed as described previously (Schwarzer et al., 1996) using a rabbit polyclonal anti-NPY antibody (1:2000; Chemicon, Temecula, CA). Immunoreactivity was tested by the avidin-biotin-peroxidase technique (Vectastain $\mathrm{ABC}$ kit; Vector Laboratories, Burlingame, CA) and then developed by incubation with $3^{\prime}, 3^{\prime}$ diaminobenzidine (Sigma, St. Louis, MO).

In in situ hybridization and immunohistochemical procedures, processing of brain sections taken at the same anteroposterior level from NPY-overexpressing and control rats was done simultaneously to allow direct comparison between the experimental groups.

Statistical analysis. Data are presented as means \pm SEM. One-way ANOVA followed by Tukey's test was used to determine the effects of vector treatment versus AAV-NSE-empty controls.

\section{Results}

\section{rAAV-NSE-NPY gene expression}

Local injection of either rAAV-NSE-NPY construct (serotype 2 or serotype $1 / 2$ ) or empty viral vector in naive rats caused similar, only minor local neuronal damage restricted to the area adjacent to the needle tract, as assessed $3 \mathrm{~d}$ and 8 weeks after vector injection by Fluoro-Jade (data not shown) or Nissl staining analysis of brain sections (Fig. 1e-h) (Xu et al., 2001). Histochemical analysis of inflammation markers such as interleukin- $1 \beta$ and the microglia marker OX-42 (complement receptor type 3) did not reveal any increase in immunostaining in the injected hippocampus or at distal sites around it (data not shown).

NPY gene overexpression in rAAV vector-injected rats was restricted to the infused hippocampus and never occurred in other extrahippocampal regions; NPY overexpression extended for $\sim 1.5 \mathrm{~mm}$ around the injection site for serotype 2 (Fig. 1a1-a5) and for $\sim 2.5 \mathrm{~mm}$ for serotype $1 / 2$ (Fig. 1b1-b5,c1-c5), as judged by evaluating the area of increased NPY mRNA expression.

Similar spread of the transduced gene was obtained by confocal microscope analysis of coronal brain sections from rats injected with rAAV-NSE carrying GFP (Figs. $2 m-o, 3 o-q$ ).

NPY gene expression induced by either vector was restricted to neurons, was maximal after 2 weeks, and was maintained for at least 3 months (present study) (Xu et al., 2001).

In rats overexpressing GFP or a different gene, such as galanin, we did not observe changes in endogenous NPY expression (data not shown), indicating that viral overexpression of a transgene 

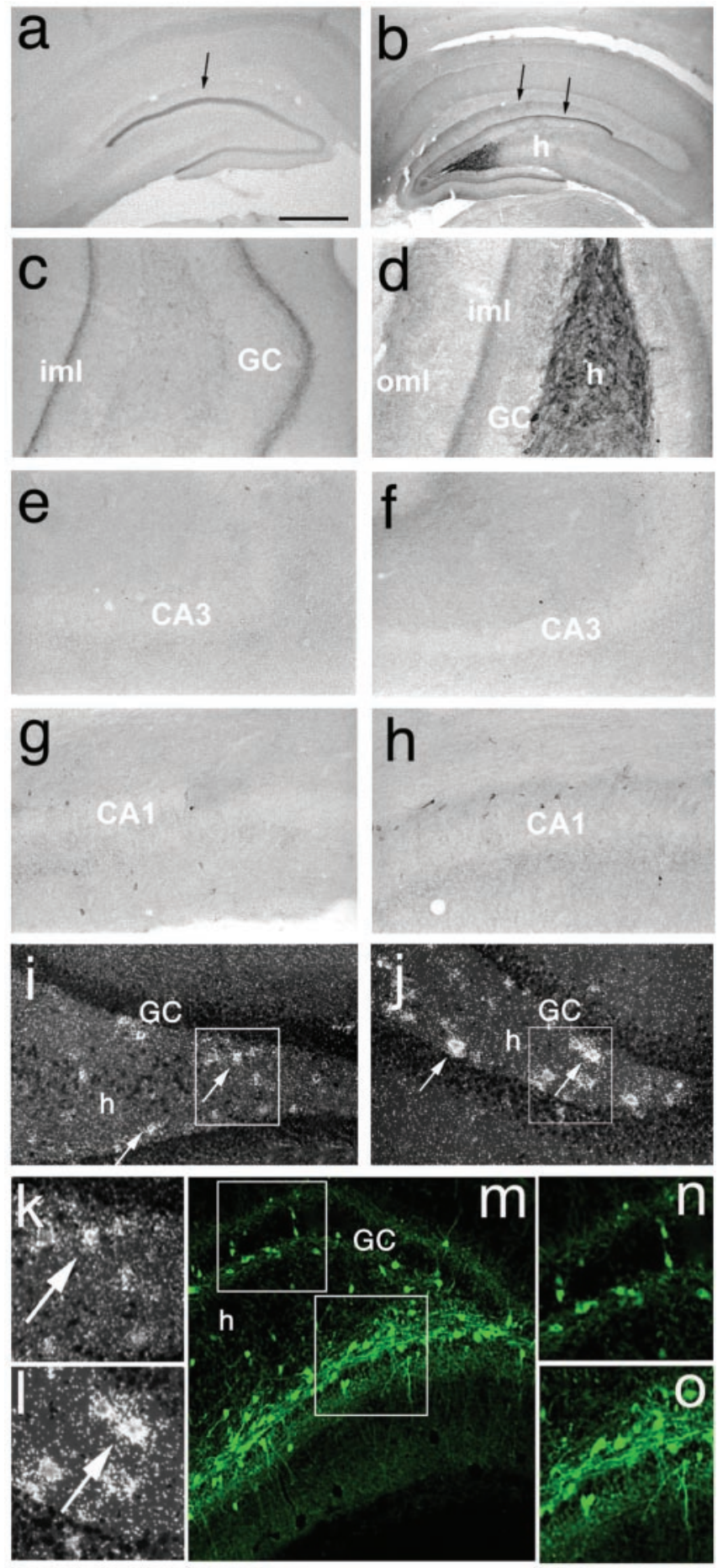

Figure 2. Expression of NPY in the injected $(b, d, f, h, j, l)$ and contralateral $(a, c, e, g, i, k)$ hippocampus of a representative rat injected 8 weeks previously with serotype 2 rAAV-NSENPY or rAAV-NSE-GFP $(k-m)$. $b$ shows enhanced NPY immunoreactivity in fibers in the hilus of the injected hippocampus and in the inner and outer molecular layers where hilar interneurons project ( $b$, arrows). A sharp band of NPY immunoreactivity is seen in the inner molecular layer contralateral to the injection site where commissural fibers originating from hilar mossy cells terminate ( $a$, arrow). Except for NPY staining in the inner molecular layer, peptide expression in the contralateral hippocampus was similar to that observed in empty vector-injected rats (data not shown). $e-h$ show high magnification of corresponding areas in $b$ and $a$, respectively. $f$ and $h$ show a lack of NPY staining in CA3 pyramidal neurons, mossy fiber terminal fields, and CA1 pyramidal cells, although stratum oriens and radiatum interneurons are visible; this was similar to what was observed in $e$ and $g$ from the contralateral hippocampus and in empty vectorinjected rats (data not shown). $i$ and $j$ show dark-field photomicrographs of hilar interneurons from slices hybridized with the ${ }^{35}$ S-labeled NPY oligoprobe. Note the enhanced NPY mRNA does not compromise the ability of the cell to translate its native peptide.

Eight weeks after vector injection, serotype 2 rAAV-NSENPY induced a significant increase in the NPY mRNA hybridization signal restricted to hilar interneurons of the injected dorsal hippocampus (Figs. 1a1-a5, 2j,l) compared with corresponding sections of rAAV-NSE-empty-injected rats (Figs. $1 d 1-d 5,2 i, k$ ).

Quantification of the hybridization signal on individual hilar interneurons by a silver grain count in digital images (Masseroli et al., 1993) showed a significant increase of 50\% in hippocampal slices of serotype 2 rAAV-NSE-NPY-injected rats compared with matched control slices [empty vector, $0.4 \pm 0.04$ grains/ $\mu \mathrm{m}^{2}(n=3$ rats $) ; \mathrm{rAAV}$-NSE-NPY, $0.6 \pm 0.07$ grains $/ \mu \mathrm{m}^{2}(n=$ 4 rats); $p<0.02]$.

Accordingly, immunohistochemical analysis of coronal brain sections encompassing the injected site (dorsal dentate gyrus) showed enhanced NPY immunoreactivity in hilar interneurons, including intensely labeled fibers in the hilus (Fig. $2 b, d$ ) and their terminal projection area in the outer molecular layer (Fig. $2 b, d$ ). NPY immunoreactivity was increased in the inner molecular layer, both in the injected and contralateral hippocampus (Fig. $2 a-d$ ), outlining the region where associational-commissural fibers, arising from mossy cells of the injected dentate hilus, terminate (Laurberg, 1979). No NPY immunoreactivity was observed in CA1 or CA3 pyramidal cells (Fig. $2 f, h$ ) or in granule neurons and their mossy fibers (Fig. $2 b, d, f$ ) in the injected hippocampus.

Our results are at variance with those reported by Xu et al. (2001) when assessing GFP overexpression in CA1 using serotype 2 vectors. Thus, these authors reported limited GFP overexpression in this hippocampal sector, whereas we never observed this phenomenon after serotype 2 virus delivery. Although the reason for this difference is unclear, we suspect that leakage of the vector solution from the injection needle during either its insertion or its withdrawal may be responsible for nonspecific CAl gene transduction in pyramidal cells located in close apposition to the injection track.

Under the same experimental conditions described above, serotype 1/2 rAAV-NSE-NPY-injected rats displayed high expression of NPY mRNA in hilar interneurons and granule cells (Fig. $3 k$ ) as well as in CA1-CA3 pyramidal cells (Fig. $3 l, m$ ) of the injected site. Strong transcript signal was also found in the subiculum (Fig. 3n). Accordingly, immunohistochemical analysis of NPY in the injected hippocampus of chimeric serotype rAAVNSE-NPY-treated rats showed strongly increased peptide levels in the terminal field of mossy fibers (Fig. $3 b, d, f$ ) and CA1 pyramidal cells (Fig. 3h). Hilar interneurons (Fig. 3d), CA3 pyramidal cells (Fig. 3f), and the subiculum (Fig. 3j) showed enhanced immunoreactivity. Increased NPY immunoreactivity was also found in the inner molecular layer of the contralateral hippocampus (Fig. 3a,c) and in both the inner and outer molecular layers of the injected hippocampus (Fig. 3b,d).

\section{$\leftarrow$}

transcript level in the polymorphic cell layer ( $j$ ) compared with the corresponding control section from empty vector-injected rats $(i) . k$ and $/$ represent enlargements of the corresponding boxed areas in $i$ and $j$. The granular signal over the granule cell layer in $j$ represents background staining. $m-0$ depict GFP distribution in interneurons in the dorsal-injected hippocampus 8 weeks after rAAV-NSE-GFP infusion. $n$ and $o$ show high magnification of boxed areas in $m$. The spotty expression of GFP in the granule cell layer in $m$ was observed sporadically and was attributable to cell bodies likely representing GABA-containing pyramidal-shaped basket cells laying close to or within the granule cell layer (Ribak and Seress, 1983). GC, Granule cell; h, hilus; CA1 and CA3, pyramidal cells; iml, inner molecular layer; oml, outer molecular layer. Scale bars: $a, b, 500 \mu \mathrm{m} ; c-j, m, 200 \mu \mathrm{m} ; k, l, n, 0,50 \mu \mathrm{m}$. 

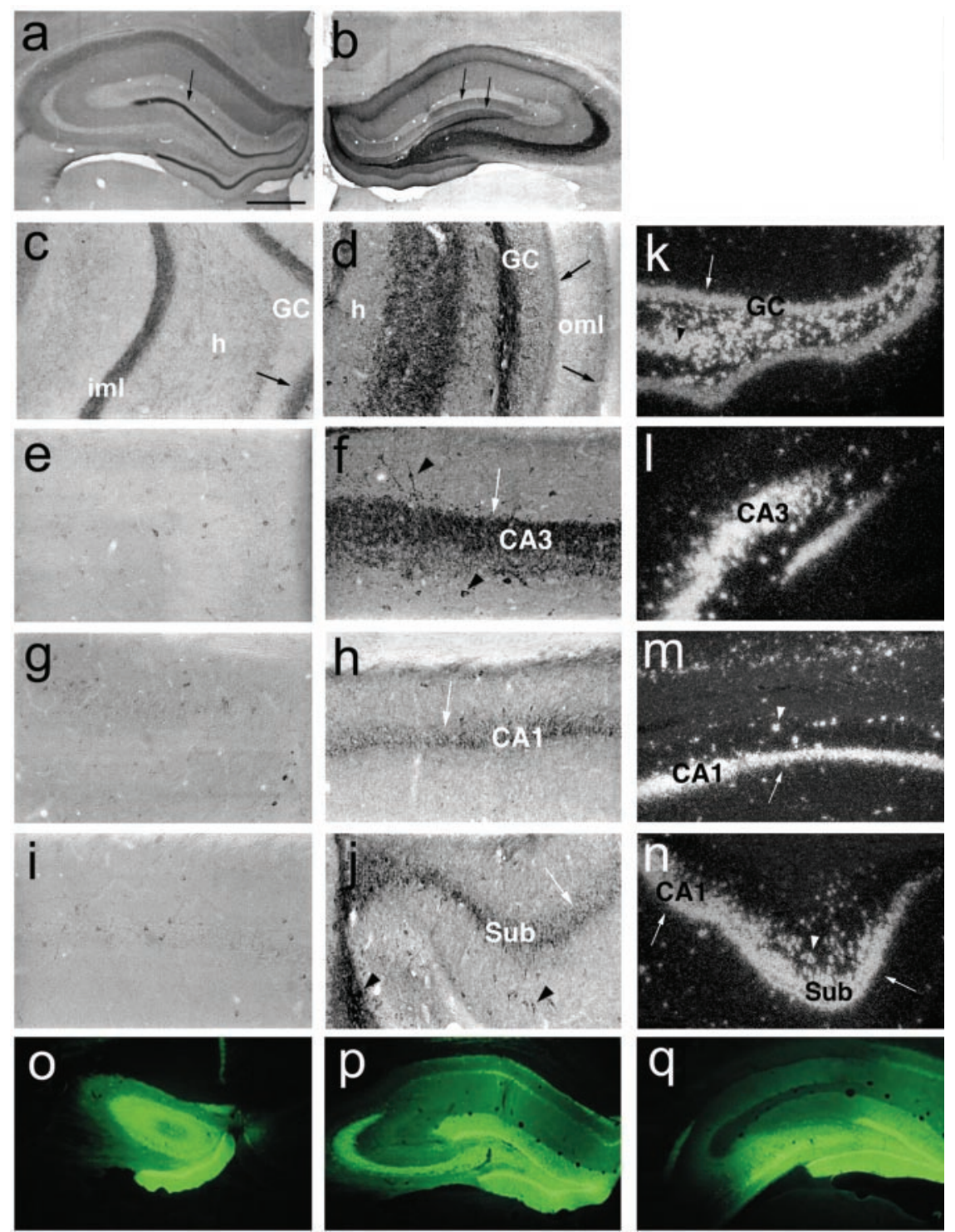

Figure 3. Expression of NPY in the injected hippocampus of a representative rat 8 weeks after infusion of serotype $1 / 2$ rAAV-NSE-NPY.a-jdepict immunocytochemical distribution of NPY. NPY immunoreactivity was predominantly expressed in the mossy fibers of the injected dorsal hippocampus $(b, d, f)$. It was present in the inner and outer molecular layers of the injected hippocampus ( $b, d$, arrows) and forms a dense immunoreactive band in the inner molecular layer of the contralateral hippocampus ( $a, c$, arrow), reflecting NPY contained in terminals of commissural fibers presumably arising from excitatory mossy cells near the injection site. NPY was also increased in CA3 ( $f$ ) and CA1 $(h)$ pyramidal neurons and in the subiculum $(j) . e, g$, and $i$ represent sections of the contralateral hippocampus corresponding to $f, h$, and $j$, respectively. NPY staining in these sections was similar to that observed in empty vector-injected rats (data not shown). $k-n$ depict dark-field photomicrographs of NPY mRNA in coronal sections of the injected hippocampus hybridized with ${ }^{35}$ S-labeled NPY oligoprobe; an intense hybridization signal is observed in granule cells $(k$, arrow), hilar interneurons $(k), C A 3(I), C A 1(m)$, and the subiculum ( $n$, arrows). Arrowheads in the various panels depict labeled interneurons. 0 - $q$ show the anteroposterior pattern of distribution of GFP in the injected dorsal hippocampus. GC, Granule cells; h, hilus; CA1 and CA3, pyramidal cells; iml, inner molecular layer; oml, outer molecular layer; Sub, subiculum. The arrow in $a$ depicts the inner molecular layer enlarged in $c$; the arrows in $b$ depict inner and outer molecular layers. Scale bars: $a, b$, $0-q, 500 \mu \mathrm{m} ; c-n, 200 \mu \mathrm{m}$.

Our unpublished data using GFP-expressing vectors also showed that in the hippocampus, the chimeric virus (AAV1/2) has higher transduction efficiency than AAV2 [similar to that reported for AAV1 by Passini et al. (2003)].

\section{Kainic acid-induced seizures}

Preliminary experiments showed that 40 and $250 \mathrm{ng}$ of kainic acid injected unilaterally into the dorsal hippocampus or into the lateral ventricle, respectively, were the lowest convulsant doses causing reproducible EEG seizures in $100 \%$ of rats without mortality.

After either intrahippocampal or intracerebroventricular injection of kainic acid, all control rats developed discrete episodes of EEG seizures lasting $2.5 \mathrm{~min}$ on average (Fig. 4b,e) (Vezzani et al., 1999b). These discrete episodes were typically associated with behavioral manifestation of "wet dog shakes" and occasional retraction of a forelimb. EEG status epilepticus, defined by continuous EEG seizure activity lasting for at least $30 \mathrm{~min}$, was observed only after intracerebroventricular application of kainic acid (Fig. 4c) and was associated with generalized behavioral clonic convulsions resembling stage 4 or 5 of kindling.

\section{Intrahippocampal kainic acid}

Both rAAV-NSE-NPY vectors significantly reduced EEG seizure activity induced by intrahippocampal application of $40 \mathrm{ng}$ of kainic acid (Fig. 5). Thus, the number of EEG seizures and their duration was decreased by $\sim 50 \%$ in serotype 2 rAAV-NSE-NPY-injected rats $(n=5)$ and by $\sim 75 \%$ in serotype $1 / 2$ rAAV-NSENPY-injected rats $(n=10 ; p<0.005$ compared with serotype 2 AAV-NSE-NPY); the time to seizure onset was delayed by approximately twofold in both groups $(p<0.01$ vs rAAV-empty; $n=10)$.

\section{Intracerebroventricular kainic acid}

Because the serotype 1/2 rAAV-NSE-NPY vector induced greater seizure reduction, additional characterization of seizure susceptibility was performed on rats injected with this chimeric vector.

The table annexed to Figure 4 shows the quantitative evaluation of EEG seizure activity induced in rats by intracerebroventricular administration of $250 \mathrm{ng}$ of kainic acid 8 weeks after intrahippocampal injection of rAAV-NSE-empty (control rats, $n=10$ ) versus serotype $1 / 2 \mathrm{rAAV}-$ NSE-NPY $(n=6)$. rAAV-NSE-NPYinjected rats had an approximately twofold delay in seizure onset and up to $~ 76 \%$ reduction in total time spent in EEG seizures $(p<0.01)$ because of abolition of EEG status epilepticus (Fig. $4 c$ ); these rats did not show generalized behavioral clonic seizures (see Materials and Methods), whereas wet dog shakes were still associated with discrete seizure episodes.

\section{Kindling-induced epileptogenesis}

Development of kindling epileptogenesis was studied in rats injected 8 weeks before with rAAV-NSE-NPY serotype 1/2 vector compared with rats injected with the empty-vector cassette.

The threshold current for inducing afterdischarge in the stimulated hippocampus before the beginning of kindling was in- 
creased by $40 \%$ in rAAV-NSE-NPY rats $(p<0.05)$ (Table 1). This parameter was not modified in rats injected with serotype 2 rAAV-NSE-NPY vector (data not shown). The number of stimuli to induce preconvulsive stages 1 and 2 was not affected by NPY overexpression, whereas the number of electrical stimuli needed to induce stage 3 (clonic contraction of back muscles) and stages 4-5 (generalized clonic motor seizures with or without loss of posture) was increased by approximately twofold ( $p<0.05$ and 0.01 ). The total number of stage $4-5$ seizures was lower in rAAV-NSE-NPY-injected rats $(4 \pm 1 ; n=7 ; p<0.01)$ compared with empty vector $(9 \pm 2 ; n=9)$. The average duration of the cumulative afterdischarge occurring during kindling in the stimulated hippocampus was reduced by $\sim 30 \%$ $(p<0.05)$.

\section{Discussion}

We have shown that in vivo overexpression of NPY in the hippocampus induced by local application of AAV-based vectors provides significant protection from limbic seizures and impairs epileptogenesis in rats. Seizure inhibition has also been described recently in rats using AAV vectors carrying the galanin gene (Haberman et al., 2003; Lin et al., 2003).

NPY gene transduction was observed exclusively in neurons because of strong neuronal tropism of these vectors.

Under normal physiological conditions, NPY is present in interneurons in the hilus, stratum oriens CA1-CA3, and radiatum CA3 (Milner et al., 1997). We observed two different patterns of NPY expression in the injected hippocampus, depending on the vector serotype: robust expression in hilar interneurons (serotype 2 ) versus additional expression in pyramidal and granule cells (chimeric serotype 1/2).

It is likely that a major part of transduced prepro-NPY is processed in a conventional way through the regulated secretory pathway into synaptic vesicles by prohormone convertases (Bellmann et al., 1991; Day et al., 1992). Expression of prohormone convertases is indeed upregulated in the brain after kainic acidinduced seizures (Marcinkiewicz et al., 1996). A minor portion (presumably $<15 \%$ ) of pro-NPY could also "spill over" into the constitutive pathway, resulting in the formation of some inactive pro-NPY not released by exocytosis (Burgess and Kelly, 1987). However, the robust pharmacological effects seen in our experiments are in favor of exocytotic release of newly formed NPY.

In the hippocampus injected with serotype 2 or serotype $1 / 2$ vectors, NPY was strongly expressed in fibers located in the inner and outer molecular layers of the dentate gyrus, and after injection of serotype $1 / 2$, NPY was also present in the terminal field of mossy fibers. In the hippocampus contralateral to that injected with serotype 2 or serotype $1 / 2$ vectors, increased NPY immunoreactivity was specifically found in the inner molecular layer where the commissural fibers terminate. This demonstrates that NPY is efficiently transported to nerve terminals within the in-

\section{rAAV-NSE-Empty}

b $20 \mathrm{~min}$

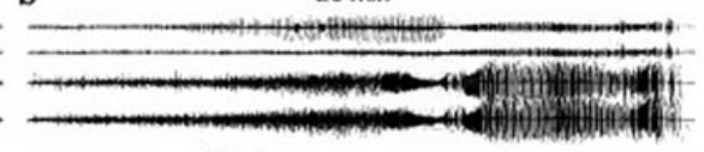

$60 \mathrm{~min}$
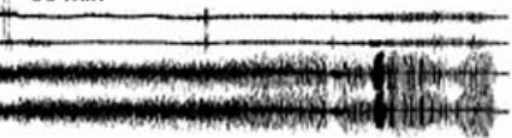

rAAV-NSE-NPY

d
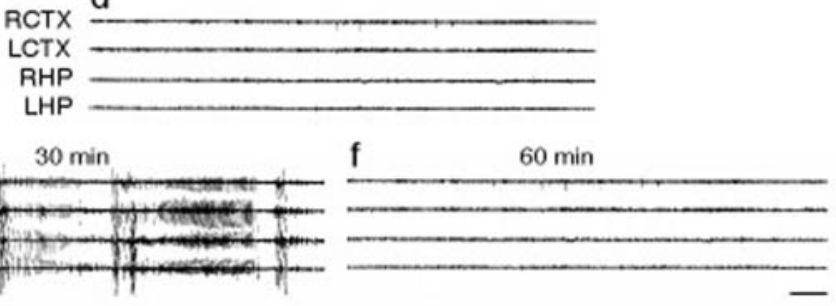

Seizure activity in rats overexpressing NPY in the hippocampus 8 weeks after rAAV-NSE- NPY injection

\begin{tabular}{lccccc}
\hline Treatment & $\begin{array}{c}\text { Onset } \\
(\mathrm{min})\end{array}$ & $\begin{array}{c}\text { Number of } \\
\text { seizures }\end{array}$ & $\begin{array}{c}\text { Time in discrete } \\
\text { seizures }(\mathrm{min})\end{array}$ & $\begin{array}{c}\text { Time in status } \\
\text { epilepticus }(\mathrm{min})\end{array}$ & $\begin{array}{c}\text { Total time in } \\
\text { seizures (min) }\end{array}$ \\
\hline rAAV-NSE-Empty & $6.2 \pm 0.3$ & $18.0 \pm 1.0$ & $53.5 \pm 6.0$ & $86.9 \pm 10.1$ & $137.0 \pm 7.9$ \\
$\begin{array}{l}\text { rAAV-NSE-NPY } \\
\text { (Serotype 1/2) }\end{array}$ & $11.5 \pm 1.8 *$ & $23.0 \pm 6.0$ & $53.4 \pm 9.7$ & 0 & $53.4 \pm 9.7^{*}$ \\
\hline
\end{tabular}

Data are the mean \pm SE ( $n=-6-10)$. Kainic acid was injected intracerebroventriculariy (250 ng in $0.5 \mu), 8$ weeks

Figure 4. EEG tracing depicting seizure activity induced by $250 \mathrm{ng}$ of intracerebroventricular kainic acid in rats injected 8 weeks previously with $\mathrm{rAAV}-\mathrm{NSE}-\mathrm{NPY}$ serotype $1 / 2(d, f)$ or empty vector $(a-c)$. $a$ and $d$ represent baseline recordings in the right cortex as described in Materials and Methods.

jected and contralateral hippocampus; thus, it may exert its action at specific release sites.

Consistent with the anticonvulsant and antiepileptogenic actions of NPY (Smialowska et al., 1996; Baraban et al., 1997; Woldbye, 1998; Marsh et al., 1999; Vezzani et al., 1999a; DePrato Primeaux et al., 2000; Reibel et al., 2001; Husum et al., 2002; Mazarati and Wasterlain, 2002), vector-mediated overexpression of this peptide significantly reduced seizure activity induced by intracerebral kainate application. In particular, rats with widespread NPY overexpression (serotype 1/2 vector) displayed greater reduction of discrete seizure episodes (i.e., 75\% compared with a 50\% decrease observed in rats with NPY overexpression restricted to hilar interneurons). Because we did not quantify the level of expression of NPY in hilar interneurons in serotype 1/2versus serotype 2-injected rats, we cannot exclude the possibility that more efficient gene transduction in this specific neuronal population rather than the more widespread expression of NPY in principal neurons by chimeric serotype is mainly responsible for greater seizure inhibition. However, our results strongly suggest that NPY expression may efficiently control hippocampal hyperexcitability at multiple synaptic sites, including hilar interneurons, mossy fibers, and CA1 pyramidal and subicular neurons connecting the hippocampus with other limbic regions (Amaral and Witter, 1995).

Prolonged EEG seizure episodes (status epilepticus) were typ- 


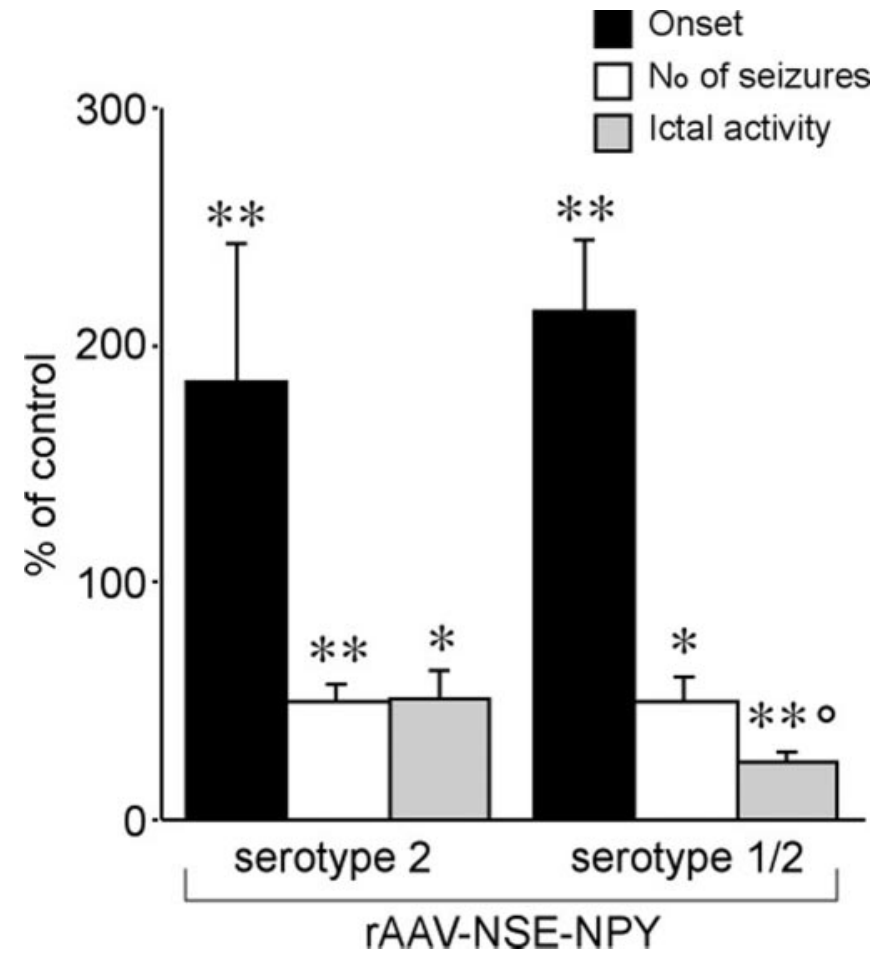

Figure 5. Inhibition of intrahippocampal kainate-induced seizures in serotype 2 versus serotype 1/2 rAAV-NSE-NPY-injected rats. Error bars indicate means \pm SE $(n=5-10)$ of the number of seizures, time spent in seizures (ictal activity), and time to onset of first seizure expressed as a percentage of values in respective controls (rats injected with rAAV-NSE-empty: onset, $6.5 \pm 0.3 \mathrm{~min}$; number of seizures, $28 \pm 5$; time in seizures, $92.8 \pm 14.3 \mathrm{~min} ; n=10$ ). Rats were injected with the respective vectors bilaterally into the dorsal hippocampus, and seizures were induced 8 weeks later by unilateral intrahippocampal application of $40 \mathrm{ng}$ of kainic acid. ${ }^{*} p<0.05 ;{ }^{* *} p<0.01$ versus respective controls; ${ }^{\circ} p<0.05$ versus serotype 2 by Tukey's test.

Table 1. Kindling epileptogenesis in rAAV-NSE-NPY-injected rats versus rAAVNSE-empty

\begin{tabular}{lcc}
\hline & Empty vector & rAAV-NSE-NPY \\
\hline Afterdischarge threshold $(\mu \mathrm{A})$ & $127 \pm 4$ & $180 \pm 21^{*}$ \\
Number of electrical stimuli & & \\
$\quad$ Stage 1 & $1.1 \pm 0.1$ & $1.3 \pm 0.1$ \\
Stage 2 & $1.4 \pm 0.3$ & $2.4 \pm 0.7$ \\
Stage 3 & $6.2 \pm 1.8$ & $12.8 \pm 2.1^{*}$ \\
Stages 4-5 & $11.4 \pm 2.5$ & $25.5 \pm 4.2^{* *}$ \\
Cumulative afterdischarge $(\mathrm{min})$ & $14.9 \pm 2.0$ & $9.9 \pm 1.0^{*}$ \\
\hline
\end{tabular}

Data are the means \pm SE of kindling parameters ( $n=7-9$ rats). Afterdischarge threshold represents the minimum current (in microamperes) inducing afterdischarge in the stimulated hippocampus before the beginning of kindling. The numbers of electrical stimuli required for inducing the respective kindling stages are reported. Cumulative afterdischarge represents the average of the sum of all afterdischarge recorded by EEG after electrical stimulation during kindling. ${ }^{*} p<0.05 ;{ }^{* *} p<0.01$ by Tukey's test.

ically observed in the hippocampi of rats injected with intracerebroventricular kainate. These episodes were associated with generalized behavioral clonic seizures, likely representing reverberant activity within the limbic circuit. Both EEG status epilepticus and behavioral clonic seizures were abolished in rats overexpressing NPY in multiple hippocampal subfields. Similarly, serotype $1 / 2$ virus-mediated NPY overexpression also effectively delayed kindling epileptogenesis, and this effect was apparent from stage 3 to stages 4-5, when generalized motor convulsions occur. Peptide overexpression may impair seizure generalization by enhancing the inhibitory tone within the hippocampus. Accordingly, the threshold current for afterdischarge induction in the hippocampus was significantly raised in rats overexpressing NPY. Inhibition of generalized seizures may also be consequent to an increase in NPY release in target areas of hippocampal efferent projections (Amaral and Witter, 1995).

Our data are in agreement with pharmacological evidence showing that subchronic NPY infusion in the hippocampus retards kindling acquisition (Reibel et al., 2000). The same inhibitory profile on kindling was found in transgenic rats selectively overexpressing NPY in CA1 pyramidal neurons and in the subiculum (Vezzani et al., 2002).

One likely mechanism underlying NPY-mediated reduction of seizures is related to the ability of the peptide to significantly suppress depolarization-induced glutamate release from mossy fibers and Schaffer collaterals. NPY overexpression in epileptic tissue occurs at sites intimately involved in seizure generation, and presynaptic NPY receptors are expressed on terminals of principal neurons and in the subiculum, where they may play a role in the anticonvulsant action of NPY by mediating inhibition of glutamate release (Colmers and Bleakman, 1994; Greber et al., 1994). Thus, overexpression of NPY may represent one of the endogenous protective mechanisms against hyperexcitability induced in the epileptic tissue. These mechanisms may be able to reduce seizure frequency or intensity but obviously cannot prevent seizure recurrence. In accordance with an endogenous anticonvulsant role of NPY, mice lacking the NPY gene have enhanced susceptibility to kainic acid-induced seizures, leading to increased mortality rate and sporadic spontaneous epileptic events (Baraban et al., 1997). Besides the possibility that endogenous protective mechanisms may fail because of preponderant excitatory drive (e.g., caused by neuronal damage, synaptic rearrangements, and plasticity), additional factors could limit the protective effects of endogenously overexpressed NPY: (1) The loss of NPY-producing cells in the epileptic sclerotic hippocampus may detract an important source of peptide release. If this is critical, then the ability to overexpress NPY in the remaining neuronal populations as well as in granule cells overexpressing Y2 receptors (Furtinger et al., 2001) using viral vectors may counteract this deficiency. (2) Exhaustion of the storage vesicles of the neuropeptide may occur during prolonged and sustained epileptic activity, thereby impairing NPY release (Bellmann et al., 1991). Again, the introduction of extra copies of the transgene may serve to supply enough peptide during prolonged epileptic activity.

Despite pronounced loss of NPY interneurons in the hilar polymorphic cell layer after severe seizures in experimental models and humans, NPY-GABA-containing subgranular interneurons are relatively well spared, as are granule cells of the dentate gyrus and also pyramidal cells in some instances (de Lanerolle et al., 1989; Sloviter, 1991; Sperk et al., 1992; Mathern et al., 1995; Furtinger et al., 2001; Sundstrom et al., 2001). Thus, surviving neurons within the sclerotic temporal lobe could represent an attractive target population for vector-mediated NPY overexpression. Chronic overexpression of NPY in the rat hippocampus does not significantly affect NPY-Y2 receptors (present study, data not shown) (Thorsell et al., 2000; Vezzani et al., 2002), and there is solid evidence for widespread and pronounced upregulation of hippocampal NPY-Y2 receptors in various animal models of epilepsy and in patients with TLE (Gobbi et al., 1998; Schwarzer et al., 1998; Furtinger et al., 2001). This indicates that one crucial receptor target mediating the inhibitory effects of NPY on glutamatergic function, and thus on neuronal excitability, is readily available to NPY in sclerotic epileptic tissue.

Although the inhibitory effects mediated by NPY overexpression in the hippocampus by viral vectors provide a proof of prin- 
ciple for their potential use to enhance endogenous transduction of "therapeutic genes," additional studies of gene transfer in spontaneously epileptic rats, and thus in pathological brain tissue, are mandatory to better assess the relevance of our finding for human epilepsy.

\section{References}

Amaral DG, Witter MP (1995) Hippocampal formation. In: Hippocampus (Paxinos G, ed), pp 443-493. New York: Academic.

Baraban SC, Hollopeter G, Erickson JC, Schwartzkroin PA, Palmiter RD (1997) Knock-out mice reveal a critical antiepileptic role for neuropeptide Y. J Neurosci 17:8927-8936.

Bellmann R, Widmann R, Olenik C, Meyer DK, Maas D, Marksteiner J, Sperk G (1991) Enhanced rate of expression and biosynthesis of neuropeptide $\mathrm{Y}$ after kainic acid-induced seizures. J Neurochem 56:525-530.

Bregola G, Dumont Y, Fournier A, Zucchini S, Quirion R, Simonato M (2000) Decreased levels of neuropeptide Y5 receptor binding sites in two experimental models of epilepsy. Neuroscience 98:697-703.

Burgess TL, Kelly RB (1987) Constitutive and regulated secretion of proteins. Annu Rev Cell Biol 3:243-293.

Clark KR, Sferra TJ, Lo W, Qu G, Chen R, Johnson PR (1999) Gene transfer into the CNS using recombinant adeno-associated virus: analysis of vector DNA forms resulting in sustained expression. J Drug Target $7: 269-283$.

Colmers WF, Bleakman D (1994) Effects of neuropeptide Y on the electrical properties of neurons. Trends Neurosci 17:373-379.

Day R, Schafer MK, Watson SJ, Chretien M, Seidah NG (1992) Distribution and regulation of the prohormone convertases $\mathrm{PC} 1$ and $\mathrm{PC} 2$ in the rat pituitary. Mol Endocrinol 6:485-497.

de Lanerolle NC, Kim JH, Robbins RJ, Spencer DD (1989) Hippocampal interneuron loss and plasticity in human temporal lobe epilepsy. Brain Res 495:387-395.

DePrato Primeaux S, Holmes PV, Martin RJ, Dean RG, Edwards GL (2000) Experimentally induced attenuation of neuropeptide-Y gene expression in transgenic mice increases mortality rate following seizures. Neurosci Lett 287:61-64.

During MJ (1997) Adeno-associated virus as a gene delivery system. Adv Drug Deliv Rev 27:83-94.

During MJ, Young D, Baer K, Lawlor P, Klugmann M (2003) Development and optimization of adeno-associated virus vector transfer into the central nervous system. Methods Mol Med 76:221-236.

El Bahh B, Herzog HH, Beck-Sickinger AG, Doods H, Colmers WF (2002) $\mathrm{Y} 2$ receptors are necessary and sufficient for anticonvulsant presynaptic action of NPY in rat and mouse hippocampus. Soc Neurosci Abstr 28:148.12.

Furtinger S, Pirker S, Czech T, Baumgartner C, Ransmayr G, Sperk G (2001) Plasticity of $\mathrm{Y} 1$ and $\mathrm{Y} 2$ receptors and neuropeptide $\mathrm{Y}$ fibers in patients with temporal lobe epilepsy. J Neurosci 21:5804-5812.

Gobbi M, Gariboldi M, Piwko C, Hoyer D, Sperk G, Vezzani A (1998) Distinct changes in peptide YY binding to, and mRNA levels of, Y1 and Y2 receptors in the rat hippocampus associated with kindling epileptogenesis. J Neurochem 70:1615-1622.

Greber S, Schwarzer C, Sperk G (1994) Neuropeptide Y inhibits potassiumstimulated glutamate release through Y2 receptors in rat hippocampal slices in vitro. Br J Pharmacol 113:737-740.

Gruber B, Greber S, Rupp E, Sperk G (1994) Differential NPY mRNA expression in granule cells and interneurons of the rat dentate gyrus after kainic acid injection. Hippocampus 4:474-482.

Haberman RP, Samulski RJ, McCown TJ (2003) Attenuation of seizures and neuronal death by adeno-associated virus vector galanin expression and secretion. Nat Med 9:1076-1080.

Husum H, Gruber SH, Bolwig TG, Mathe AA (2002) Extracellular levels of NPY in the dorsal hippocampus of freely moving rats are markedly elevated following a single electroconvulsive stimulation, irrespective of anticonvulsive Y1 receptor blockade. Neuropeptides 36:363-369.

Kaplitt MG, Leone P, Samulski RJ, Xiao X, Pfaff DW, O’Malley KL, During MJ (1994) Long-term gene expression and phenotypic correction using adeno-associated virus vectors in the mammalian brain. Nat Genet 8:148-154.

Kopp J, Nanobashvili A, Kokaia Z, Lindvall O, Hokfelt T (1999) Differential regulation of mRNAs for neuropeptide $\mathrm{Y}$ and its receptor subtypes in widespread areas of the rat limbic system during kindling epileptogenesis. Brain Res Mol Brain Res 72:17-29.

Laurberg S (1979) Commissural and intrinsic connections of the rat hippocampus. J Comp Neurol 184:685-708.

Lin EJ, Richichi C, Young D, Baer K, Vezzani A, During MJ (2003) Recombinant AAV-mediated expression of galanin in rat hippocampus suppresses seizure development. Eur J Neurosci 18:1-6.

Marcinkiewicz M, Seidah NG, Chretien M (1996) Implications of the subtilisin/kexin-like precursor convertases in the development and function of nervous tissues. Acta Neurobiol Exp 56:287-298.

Marksteiner J, Ortler M, Bellmann R, Sperk G (1990) Neuropeptide Y biosynthesis is markedly induced in mossy fibers during temporal lobe epilepsy of the rat. Neurosci Lett 112:143-148.

Marsh DJ, Baraban SC, Hollopeter G, Palmiter RD (1999) Role of the Y5 neuropeptide Y receptor in limbic seizures. Proc Natl Acad Sci USA 96:13518-13523.

Masseroli M, Bollea A, Bendotti C, Forloni G (1993) In situ hybridization histochemistry quantification: automatic count on single cell in digital image. J Neurosci Methods 47:93-103.

Mastakov MY, Baer K, Kotin RM, During MJ (2002) Recombinant adenoassociated virus serotypes 2- and 5-mediated gene transfer in the mammalian brain: quantitative analysis of heparin co-infusion. Mol Ther 5:371-380

Mathern GW, Babb TL, Pretorius JK, Leite JP (1995) Reactive synaptogenesis and neuron densities for neuropeptide $\mathrm{Y}$, somatostatin, and glutamate decarboxylase immunoreactivity in the epileptogenic human fascia dentata. J Neurosci 15:3990-4004.

Mazarati A, Wasterlain CG (2002) Anticonvulsant effects of four neuropeptides in the rat hippocampus during self-sustaining status epilepticus. Neurosci Lett 331:123-127.

Milner TA, Wiley RG, Kurucz OS, Prince SR, Pierce JP (1997) Selective changes in hippocampal neuropeptide $\mathrm{Y}$ neurons following removal of the cholinergic septal inputs. J Comp Neurol 386:46-59.

Mitchell J, Cook I, Hervey V (1997) Effect of seizures on hippocampal peptidergic neurons. Neuropathol Appl Neurobiol 23:299-306.

Passini MA, Watson DJ, Vite CH, Landsburg DJ, Feigenbaum AL, Wolfe $\mathrm{JH}$ (2003) Intraventricular brain injection of adeno-associated virus type 1 (AAV1) in neonatal mice results in complementary patterns of neuronal transduction to AAV2 and total long-term correction of storage lesions in the brains of beta-glucuronidase-deficient mice. J Virol 77:7034-7040.

Patrylo PR, van den Pol AN, Spencer DD, Williamson A (1999) NPY inhibits glutamatergic excitation in the epileptic human dentate gyrus. J Neurophysiol 82:478-483.

Pedrazzini T, Pralong F, Grouzmann E (2003) Neuropeptide Y: the universal soldier. Cell Mol Life Sci 60:350-377.

Racine RJ (1972) Modification of seizure activity by electrical stimulation. II. Motor seizure. Electroencephalogr Clin Neurophysiol 32:281-294.

Redrobe JP, Dumont Y, St-Pierre JA, Quirion R (1999) Multiple receptors for neuropeptide $\mathrm{Y}$ in the hippocampus: putative roles in seizures and cognition. Brain Res 848:153-166.

Reibel S, Larmet Y, Carnahan J, Marescaux C, Depaulis A (2000) Endogenous control of hippocampal epileptogenesis: a molecular cascade involving brain-derived neurotrophic factor and neuropeptide Y. Epilepsia 41 [Suppl 6]:S127-S133.

Reibel S, Nadi S, Benmaamar R, Larmet Y, Carnahan J, Marescaux C, Depaulis A (2001) Neuropeptide $Y$ and epilepsy: varying effects according to seizure type and receptor activation. Peptides 22:529-539.

Ribak CE, Seress L (1983) Five types of basket cell in the hippocampal dentate gyrus: a combined Golgi and electron microscopic study. J Neurocytol 12:577-597.

Schwarzer C, Sperk G, Samanin R, Rizzi M, Gariboldi M, Vezzani A (1996) Neuropeptides-immunoreactivity and their mRNA expression in kindling: functional implications for limbic epileptogenesis. Brain Res Brain Res Rev 22:27-50.

Schwarzer C, Kofler N, Sperk G (1998) Up-regulation of neuropeptide Y-Y2 receptors in an animal model of temporal lobe epilepsy. Mol Pharmacol 53:6-13.

Sloviter RS (1991) Permanently altered hippocampal structure, excitability, and inhibition after experimental status epilepticus in the rat: the "dormant basket cell" hypothesis and its possible relevance to temporal lobe epilepsy. Hippocampus 1:41-66. 
Smialowska M, Sopala M, Tokarski K (1996) Inhibitory effect of intrahippocampal NPY injection on amphetamine-induced behavioural activity. Neuropeptides 30:67-71.

Sperk G, Marksteiner J, Gruber B, Bellmann R, Mahata M, Ortler M (1992) Functional changes in neuropeptide $\mathrm{Y}$ - and somatostatin-containing neurons induced by limbic seizures in the rat. Neuroscience 50:831-846.

Sundstrom LE, Brana C, Gatherer M, Mepham J, Rougier A (2001) Somatostatin- and neuropeptide Y-synthesizing neurones in the fascia dentata of humans with temporal lobe epilepsy. Brain 124:688-697.

Thorsell A, Michalkiewicz M, Dumont Y, Quirion R, Caberlotto L, Rimondini R, Mathe AA, Heilig M (2000) Behavioral insensitivity to restraint stress, absent fear suppression of behavior and impaired spatial learning in transgenic rats with hippocampal neuropeptide $\mathrm{Y}$ overexpression. Proc Natl Acad Sci USA 97:12852-12857.

Vezzani A, Sperk G, Colmers WF (1999a) Neuropeptide Y: emerging evidence for a functional role in seizure modulation. Trends Neurosci 22:25-30.

Vezzani A, Conti M, De Luigi A, Ravizza T, Moneta D, Marchesi F, De Simoni MG (1999b) Interleukin-1 $\beta$ immunoreactivity and microglia are en- hanced in the rat hippocampus by focal kainate application: functional evidence for enhancement of electrographic seizures. J Neurosci 19:5054-5065.

Vezzani A, Rizzi M, Conti M, Samanin R (2000) Modulatory role of neuropeptides in seizures induced in rats by stimulation of glutamate receptors. J Nutr 130 [Suppl 4]:1046S-1048S.

Vezzani A, Michalkiewicz M, Michalkiewicz T, Moneta D, Ravizza T, Richichi C, Aliprandi M, Mule F, Pirona L, Gobbi M, Schwarzer C, Sperk G (2002) Seizure susceptibility and epileptogenesis are decreased in transgenic rats overexpressing neuropeptide Y. Neuroscience 110:237-243.

Weinshenker D, Szot P, Miller NS, Rust NC, Hohmann JG, Pyati U, White SS, Palmiter RD (2001) Genetic comparison of seizure control by norepinephrine and neuropeptide Y. J Neurosci 21:7764-7769.

Woldbye DP (1998) Antiepileptic effects of NPY on pentylenetetrazole seizures. Regul Pept 75-76:279-282.

Xu R, Janson CG, Mastakov M, Lawlor P, Young D, Mouravlev A, Fitzsimons H, Choi KL, Ma H, Dragunow M, Leone P, Chen Q, Dicker B, During MJ (2001) Quantitative comparison of expression with adeno-associated virus (AAV-2) brain-specific gene cassettes. Gene Ther 8:1323-1332. 